\title{
Plinabulin, an inhibitor of tubulin polymerization, targets KRAS signaling through disruption of endosomal recycling
}

\author{
PATRICK J. CIMINO ${ }^{1}$, LAN HUANG ${ }^{2}$, LIHUA DU ${ }^{2}$, YANPING WU ${ }^{2}$, JAMIE BISHOP ${ }^{3}$, \\ JESSICA DALSING-HERNANDEZ ${ }^{4}$, KARI KOTLARCZYK $^{4}$, PAUL GONZALES ${ }^{4}$, JENNIFER CAREW $^{5}$, \\ STEFFAN NAWROCKI ${ }^{5}$, MARY ANN JORDAN ${ }^{3}$, LESLIE WILSON ${ }^{3}$, \\ G. KENNETH LLOYD ${ }^{2}$ and HANS-GEORG WIRSCHING ${ }^{6}$
}

\author{
${ }^{1}$ Department of Pathology, University of Washington, Seattle, WA 98104; ${ }^{2}$ BeyondSpring Pharmaceuticals Inc., \\ New York City, NY 10005; ${ }^{3}$ Biomolecular Science and Engineering, University of California, Santa Barbara, CA 93106; \\ ${ }^{4}$ Translational Drug Development Inc., Scottsdale, AZ 85259; ${ }^{5}$ Institute for Drug Development, University of Texas, \\ San Antonio, TX 78249, USA; ${ }^{6}$ Department of Neurology, University Hospital Zurich, CH-8091 Zurich, Switzerland
}

Received July 27, 2018; Accepted February 2, 2019

DOI: $10.3892 /$ br.2019.1196

\begin{abstract}
Constitutive activation of Kirsten rat sarcoma viral oncogene homolog (KRAS) is the most common oncogenic event in certain types of human cancer and is associated with poor patient survival. Small molecule signaling inhibitors have improved the clinical outcomes of patients with various cancer types but attempts to target KRAS have been unsuccessful. Plinabulin represents a novel class of agents that inhibit tubulin polymerization with a favorable safety profile in clinical trials. In the present study, the potency of plinabulin to inhibit tubulin polymerization and growth of KRAS-driven cancer cells was characterized. In vivo efficacy of plinabulin was tested in two different mouse models; one being the RCAS/t-va gene transfer system and the other being a xenograft model. In vitro cell culture tubulin polymerization assays were used to complement the mouse models. There was improved survival in a KRAS-driven mouse gene transfer glioma model, but lack of benefit in a similar model, without constitutively active KRAS, which supports the notion of a KRAS-specific effect. This survival benefit was mediated, at least in part, by the ability of plinabulin to inhibit tubulin polymerization and disrupt endosomal recycling. It was proposed a mechanism of compromised endosomal recycling of displaced KRAS through targeting microtubules that yields inhibition of protein kinase B, but not extracellular signal regulated kinase (ERK) signaling, therefore lending rationale
\end{abstract}

Correspondence to: Dr Hans-Georg Wirsching, Department of Neurology, University Hospital Zurich, Frauenklinikstr 26, CH-8091 Zurich, Switzerland

E-mail: hans-georg.wirsching@usz.ch

Key words: Plinabulin, Kirsten rat sarcoma viral oncogene homolog, endosome, tubulin, cancer to combination treatments of tubulin- and ERK-targeting agents in KRAS-driven cancer.

\section{Introduction}

Activating point mutations in Kirsten rat sarcoma viral oncogene homolog (KRAS) are present in $\sim 30 \%$ of human cancer, including the vast majority of types of pancreatic cancer and large fractions of lung, colon and breast cancer (1). Furthermore, elevation of the activity of KRAS and other ras family members due to upstream signaling is very common in most types of cancer (2). KRAS is a small guanosine triphosphatase (GTP-ase) that mediates survival, proliferation and cytoskeletal organization of cancer cells by transducing or enhancing down-stream signals of the dual specificity mitogen-activated protein kinase kinase mek-2 (MEK)/extracellular signaling-regulated kinase (ERK) and of the phosphoinositide 3 -kinase (PI3K)/protein kinase B (Akt) signaling cascade (3). The presence of constitutively active KRAS is associated with chemotherapy resistance and poor patient outcome, but so far attempts to directly target KRAS have failed (4). KRAS exerts its functions at the inner cellular membrane and requires continuous recycling of mislocalized protein through endosomes. The microtubule network is critically involved in this subcellular segregation and trafficking of endosomal recycling $(5,6)$. Drugs that target microtubules include taxanes and vinca alcaloids. These drugs are among the best studied, longest standing cancer drugs and act as microtubule stabilizers that slow or block mitosis at the metaphase-anaphase transition, eventually resulting in apoptosis (7). Tissue penetration of taxanes and vinca alcaloids is however limited and the therapeutic index is low.

Plinabulin (NPI-2358) is a synthetic analogue of halimide, an Aspergillus-derived natural product that targets the tubulin network through a mechanism that is distinct from that of taxanes and vinca alcaloids: Instead of stabilization of polymerized microtubules, plinabulin inhibits polymerization by interacting with the colchicine-binding domain of 
$\beta$-tubulin $(8,9)$. Drugs that target the colchicine-binding domain were historically deemed too toxic to be effective anticancer agents, but novel compounds including plinabulin are more specific and potent inhibitors of tubulin polymerization (7), and tissue penetration is more favorable compared to classic tubulin stabilizing drugs (10). Furthermore, plinabulin demonstrated a favorable safety profile and encouraging antitumor responses in two early phase clinical trials $(10,11)$. Based on these results, plinabulin is currently in phase III clinical development in combination with docetaxel and in phase I/II in combination with nivolumab for advanced non-small cell lung cancer (NCT02504489 and NCT02812667). In the present study the inhibition of tubulin polymerization by plinabulin was described and the preclinical results of the efficacy of plinabulin in a KRAS driven murine tumor models and outline effects of plinabulin on intracellular KRAS trafficking and down-stream signaling were reported.

\section{Materials and methods}

Compounds and reagents. Plinabulin was obtained from BeyondSpring Pharmaceuticals, Inc. (New York, NY, USA), irinotecan was obtained from Teva Pharmaceuticals (Irvine, CA, USA), docetaxel and paclitaxel were from Selleckchem (Houston, TX, USA). For in vivo studies, all compounds were diluted in 5\% dextrose for intraperitoneal (i.p.) or intravenous (i.v.) injection of indicated doses. For in vitro studies, all drugs were diluted in complete growth media with $1 \%$ dimethyl-sulfoxide (DMSO). In vitro EGF treatment was performed at a concentration of $100 \mathrm{ng} / \mathrm{ml}$.

Tubulin polymerization assays. Microtubule protein (MTP) preparations consisting of $70 \%$ tubulin and 30\% microtubule-associated proteins were isolated from a bovine brain, polymerized into microtubules and monitored by light scattering at $350 \mathrm{~nm}$. Transmission electron microscopy was used to determine the mean length distribution of microtubules in the absence or presence of the drug. In brief, samples were fixed at room temperature with $4 \%$ formaldehyde and $2.5 \%$ glutaraldehyde in $0.1 \mathrm{M}$ PIPES buffer for $30 \mathrm{~min}$ and stained at room temperature for $7 \mathrm{~min}$ utilizing primary antibodies and for $2 \mathrm{~min}$. with gold-labeled secondary antibodies. Embeding was performed at $60^{\circ} \mathrm{C}$ with epoxy resin. Section thickness was $60 \mathrm{~nm}$. Grids were viewed in a Jeol electron microscope-1200 EX11 (JEOL, Ltd., Tokyo, Japan) at x2,000 and $\mathrm{x} 30,000$ magnification. The Zeiss MOPIII was used to determine microtubule length distributions and mean lengths for at least 100 microtubules per sample.

Cell culture. All cell lines were obtained from the American Type Culture Collection (Manassas, VA, USA). HCT-15, A549 and MDA-MB-231 cells were maintained in RPMI-1640 medium (Lonza Group, Ltd., Basel, Switzerland). LoVo cells were maintained in F12-K medium (Corning/Cellgro; Corning Inc., Corning, NY, USA). DF1 cells were maintained in Gibco Dulbecco's modified Eagle's medium (DMEM; Thermo Fisher Scientific, Inc., Waltham, MA, USA). All cell culture media were supplemented with $10 \%$ fetal bovine serum (FBS; Avantor, Inc., Radnor, PA, USA) and housed in a $5 \% \mathrm{CO}_{2}$ atmosphere at $37^{\circ} \mathrm{C}$, except the DF1 cells which were cultured at $39^{\circ} \mathrm{C}$.

Immunocytology. A549 cells $\left(1,000\right.$ cells $\left./ \mathrm{cm}^{2}\right)$ were seeded overnight on coverslips. Cells were fixed in $4 \%$ paraformaldehyde for $10 \mathrm{~min}$ at room temperature, followed by permeabilization using $0.1 \%$ Triton in PBS for $5 \mathrm{~min}$ and blocking of unspecific binding with 5\% donkey serum (Jackson ImmunoResearch Laboratories, Inc., West Grove, PA, USA) in PBS. These steps were done at room temperature and washing with PBS was done between any steps. Primary antibodies were rabbit anti-early endosomal antigen (EEA)1 (1:300; cat. no. 610456; BD Bioscences, San Jose, CA, USA) and mouse anti-KRAS (1:100; cat. no. ab172949; Abcam, Cambridge, MA, USA) and were applied overnight at $4^{\circ} \mathrm{C}$. After additional washing steps, cells were incubated with secondary donkey anti-rabbit-488 (1:200; cat. no. 711-545-1521; Jackson ImmunoResearch Laboratories, Inc.) and anti-mouse-Cy3 (1:200; cat. no. 715-165-150; Jackson ImmunoResearch Laboratories, Inc.) antibodies in PBS with 5\% donkey serum for $30 \mathrm{~min}$ at room temperature, followed by washing and addition of Hoechst 33342 Solution $(1 \mu \mathrm{g} / \mathrm{ml}$; Thermo Fisher Scientific, Inc.) for $3 \mathrm{~min}$, followed by washing, $\mathrm{H}_{2} \mathrm{O}$ for $3 \mathrm{~min}$, $100 \%$ ethanol for $3 \mathrm{~min}$ and air-drying prior to embedding (all steps at room temperature). Images were captured with a Zeiss LSM 780 NLO confocal microscope and quantification was performed using ImageJ, version 1.51 (National Institute of Health, Bethesda, MD, USA).

In vitro cell viability assays. Indicated cell lines were grown in standard serum-containing media (RPMI with 10\% FBS from American Type Culture Collection). Cell viability was measured in triplicate utilizing the adenosine triphosphate-based CellTiter-Glo ${ }^{\circledR}$ assay (Promega Corporation, Madison, WI, USA).

Murine xenograft models. Animal studies were carried out in accordance with the Guide for the Care and Use of Laboratory Animals, as adopted by the U.S. National Institutes of Health. Animal research was prospectively approved by the Institutional Animal Care and Use Committee (Animal assurance institutional \#A3226-01; Protocol \#50842, Fred Hutchinson Cancer Research Center, Seattle, WA, USA). Female athymic Foxn1nu (BALB/c nude) mice between 4 to 6 weeks of age were obtained from Harlan Laboratories, Inc. (Madison, WI, USA). A total of 84 mice weighing 18-24 g and kept under germ-free conditions with a 12-h light/dark cycle at $20-24^{\circ} \mathrm{C}$ with $40-60 \%$ humidity. For the HCT-15 and LoVo models, mice were anesthetized by isoflurane and inoculated with $0.1 \mathrm{ml}$ of a $50 \%$ media $/ 50 \%$ Matrigel mixture containing a suspension of cultured tumor cells $\left(1 \times 10^{7}\right.$ cells/mouse for LoVo, $5 \times 10^{6}$ cells/mouse for HCT-15). As the tumors grew, the length (largest diameter) and width (smallest diameter) of tumors were measured using a digital caliper to determine the mean radius for tumor volume estimates assuming a spherical geometry and calculation per $V_{\text {tumor }}=4 / 3 \times \pi \times r^{3}$. Automated calculation and documentation of tumor volume estimates was done using the animal study management software Study Director V.2.1.1 (Studylog Systems, Inc., San Francisco, CA, USA). When tumor volume estimates exceeded $50-60 \mathrm{~mm}^{3}$, 
mice were randomized into control and treatment groups, being pair-matched by tumor size, and dosing was initiated (Day 1). Mice were euthanized as described below.

RCAS/t-va glioma models. A total of 40 male $(\mathrm{n}=23)$ or female $(\mathrm{n}=17) \mathrm{N} / \mathrm{t}-\mathrm{va} ;$ Ink4a/Arf ${ }^{-/}$agouti mice (Jackson Laboratory, Bar Harbor, ME, USA) were kept under germ-free conditions in a $12 \mathrm{~h}$ light $/ 12 \mathrm{~h}$ dark cycle at $20-24^{\circ} \mathrm{C}$ with $40-60 \%$ humidity. Mice were aged 6-8 weeks and weighed 18-24 g. These mice expressed the avian t-va receptor under control of the nestin promoter were injected intracranially with DF-1 cells (American Type Culture Collection) as hosts for the replicative avian retroviral gene transfer vector RCAS (Addgene, Inc., Cambridge, MA, USA). Tumors were generated utilizing two RCAS vectors for the expression of platelet-derived growth factor B (PDGFB) and either KRAS encoding the activating mutation KRAS ${ }^{G 12 A}$, or green fluorescent protein (GFP). DF-1 cells infected with these RCAS strains were injected into the brains of adult mice (aged 4 to 6 weeks) under the anesthetic isoflurane. A total of $1 \mu \mathrm{l}$ of a 1:1 mixture for a total combined injection of $4 \times 10^{4}$ transfected DF-1 cell suspension was delivered using a 30-gauge needle attached to a Hamilton syringe. Coordinates were bregma $0 \mathrm{~mm}$, lateral $-0.5 \mathrm{~mm}$ (right of midline) and depth $-1.5 \mathrm{~mm}$ from the dural surface. Mice were put on the study when they lost $>0.3 \mathrm{~g}$ total over 2 consecutive days or displayed outward signs of a tumor. Mice $(n=89)$ were treated with vehicle or plinabulin at $7.5 \mathrm{mg} / \mathrm{kg}$ twice per week for up to 10 weeks. The mice were euthanized in a carbon dioxide chamber (flow rate of $\mathrm{CO}_{2}$ displacing $25 \%$ of the chamber volume per minute for $5 \mathrm{~min}$ total in accordance with American Veterinary Medical Association Euthanasia Guidelines of 2013) when they lost $>20 \%$ of their body weight, displayed a lack of mobility, inability to feed or weighed $<14 \mathrm{~g}$ for a male/12 $\mathrm{g}$ for a female. Animal death was confirmed following at least 1 min of immobility within the chamber, which was followed immediately by decapitation and harvesting of the brain tissue.

Statistical analysis. All statistical comparisons were performed using GraphPad Prism 7 (GraphPad Software, Inc., San Diego, CA, USA). Kaplan-Meier survival curves were prepared using and analyzed using the log-rank (Mantel-Cox) test. Time series data were analyzed using linear regression with the mean \pm standard error calculated for each time-point. Direct comparisons were performed using a two-tailed t-test. $\mathrm{P}<0.05$ was considered to indicate a statistically significant difference. All experiments were performed in triplicate unless stated otherwise.

\section{Results}

Plinabulin inhibits microtubule polymerization. The overlapping binding sites of plinabulin and colchicine at the $\alpha / \beta$-tubulin interface led to characterizing and comparing the effects of the two drugs on microtubule dynamics. In a cell-free MTP-containing model system, plinabulin inhibited microtubule formation more potently than colchicine, as measured by turbidity spectra (Fig. 1A). The concentrations of plinabulinand colchicine at which polymerization was inhibited by $50 \%\left(\mathrm{IC}_{50}\right)$ were $2.4 \mu \mathrm{M}$ [standard deviation (SD) +/- 0.4,
$\mathrm{N}=4$ independent experiments] and 7.6 $\mu \mathrm{M}(\mathrm{SD}+/-2.4, \mathrm{~N}=3)$, respectively. The tubulin polymerization profiles obtained in the presence of different concentrations of plinabulin or colchicine differed in two ways: First, the initial rate of increase in absorbance over time decreased with increasing drug concentrations of plinabulin, indicating that there is a lag period for microtubule formation. In contrast, the initial rate of polymerization is unchanged at all concentrations of colchicine. Second, microtubule formation in the presence of plinabulin does not reach steady state at high drug concentrations, as indicated by the absorbance values that increase linearly with time, whereas in the presence of high concentrations of colchicine steady state is readily reached. These data are suggestive of a reduction of the pool of soluble, assembly-competent tubulin in the presence of plinabulin. In contrast, colchicine binds nearly irreversibly and induces a rate-limiting conformational alterations in tubulin that, once incorporated into the lattice, inhibits further microtubule polymerization at substoichiometric concentrations (12). Transmission electron microscopy was performed to further validate these results. No microtubule aggregates were identified in the presence of plinabulin $1.25 \mu \mathrm{M}$ (Fig. 1B) and $2.5 \mu \mathrm{M}$ (data not shown). The mean microtubule length was however potently reduced to $34 \%$ of the DMSO-treatment group at $1.25 \mu \mathrm{M}$ plinabulin, whereas colchicine $1.25 \mu \mathrm{M}$ reduced the mean microtubule length to $63 \%$ (Fig. 1C; Fig. S1). Next, how these results translated in a cellular system was evaluated. The $\mathrm{IC}_{50}$ of plinabulin for inhibiting mitosis in MCF-7 breast cancer cells was $17 \mathrm{nM}$ and mitosis was halted at the prometaphase (Fig. S2), suggesting that tubulin bound by plinabulin may not be assembly competent to be incorporated into microtubules.

Plinabulin synergizes with chemotherapy in KRAS-driven xenograft cancer models. In consideration to the limited activity of chemotherapy in various cancer types with constitutive KRAS activity, whether the distinct mechanism of action of plinabulin yielded antitumor activity in these types of cancer was evaluated. In a panel of 8 human cell lines derived from pancreatic, colorectal and renal cancer, plinabulin inhibited growth in the nanomolar range in all cell lines, except for the pancreatic cancer cell line HPAC (Fig. 2A). This led the present study to also investigate the putative anticancer effects of plinabulin in vivo in a panel of five KRAS mutated xenograft models. In contrast to the in vitro results, plinabulin monotherapy failed to inhibit the growth of LoVo and HCT-15 colorectal cancer (Fig. 2B; Fig. S3A) and exerted no more than a moderate effect on the growth of MDA-MB-231 breast cancer alone (Fig. 2C). The growth of the KRAS mutated DU-145 prostate cancer (Fig. 2D) and A549 lung adenocarcinoma (Fig. S3B) models was not affected by plinabulin monotreatment. However, in combination with standard chemotherapeutic agents administered in these cancers, the additive or synergistic effects of plinabulin were observed on the inhibition of tumor growth, including combinations with the topoisomerase inhibitor irinotecan and with the tubulin targeting agents, paclitaxel and docetaxel (Fig. 2; Fig. S3). These data point towards effects of plinabulin other than direct cytotoxicity and therefore led to down-stream KRAS signaling being evaluated next. 
A
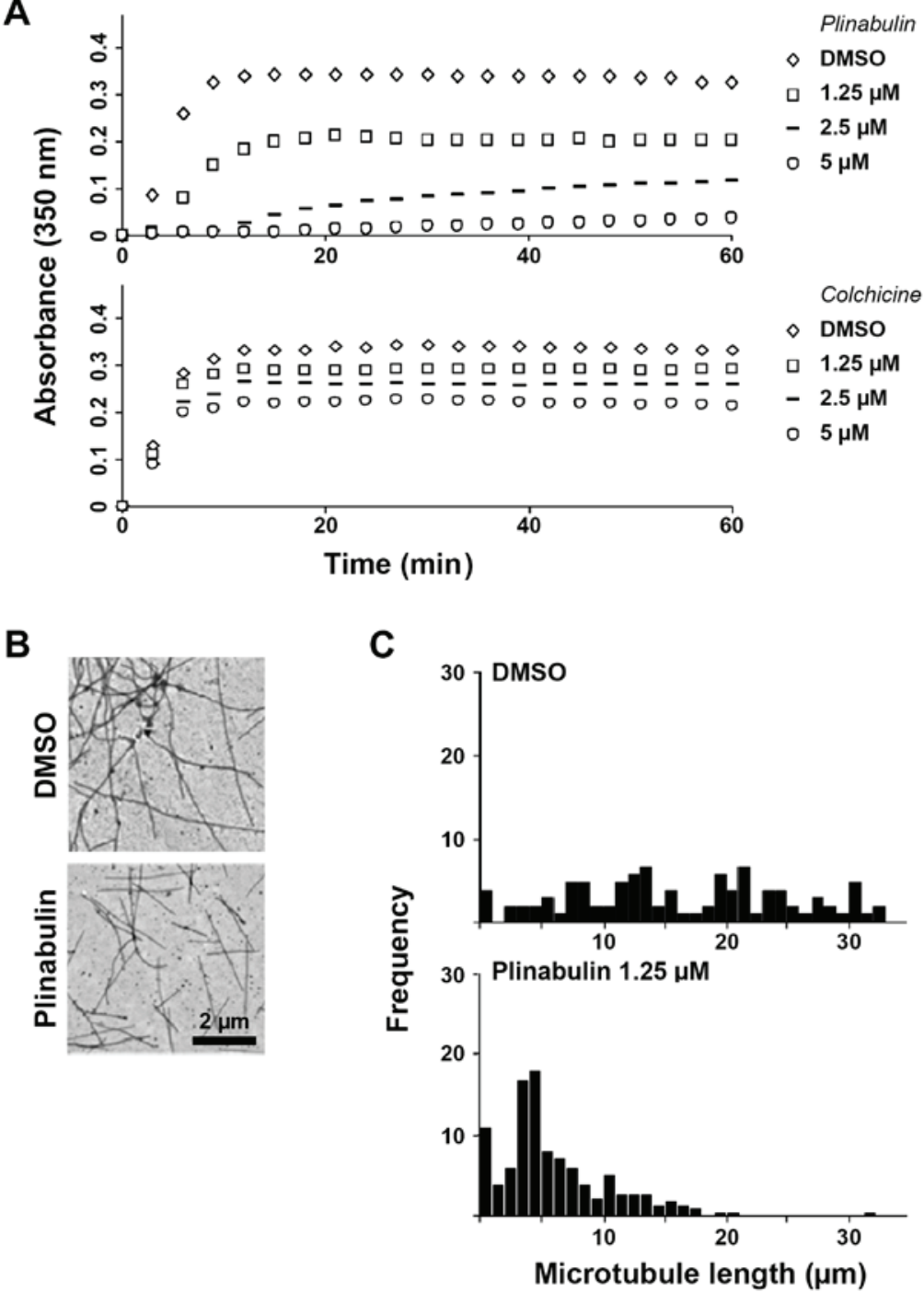

Figure 1. Plinabulin inhibits tubulin polymerization. (A) Turbidity spectra of cell-free microtubule protein polymerization in the presence of DMSO drug vehicle or indicated concentrations of plinabulin (upper panel) or colchicine (lower panel). (B) Microtubules polymerized in the presence of DMSO or $1.25 \mu \mathrm{M}$ plinabulin for 60 min prior to electron microscopy. (C) Frequency histograms of mean microtubule lengths at steady state in the presence of DMSO or plinabulin $2.5 \mu \mathrm{M}$. DMSO, dimethyl sulfoxide.

Tubulin depolymerization yields displacement of KRAS to endosomes. In non-small cell lung and other types of cancer, downstream effects of KRAS signaling include the activation of the MEK/ERK and PI3K/Akt pathways (3). Furthermore, ERK is regulated by a variety of converging pathways that are activated under cellular stress including chemotherapy with tubulin-targeting agents, whereas the key activator of Akt is the KRAS target phosphoinositide 3-kinase (PI3K) (13). The proto-oncogene phosphatase and tensin homolog (PTEN) is the key inhibitor of PI3K, therefore rendering intact PTEN a pre-requisite for KRAS-dependency of cancer cells (14). In PTEN proficient A549 lung cancer cells, disassembly of the microtubule network utilizing plinabulin concentrations in the range of its $\mathrm{IC}_{50}$ for in vitro tubulin depolymerization led to rapid accumulation of KRAS in early endosomes (mean endosomal EEA1-colocalized KRAS in DMSO vs. Plinabulin $=2$ vs. $31 \%$, $\mathrm{P}<0.001, \mathrm{~N}=5$ ) (Fig. 3A and B). Furthermore, overall KRAS levels were decreased upon plinabulin treatment, which may reflect lysosomal degradation, but this needs to be investigated further. The two key down-stream effectors of KRAS signaling,
ERK and Akt, were affected differently by plinabulin treatment and subsequent KRAS depletion: ERK phosphorylation was increased, but epidermal growth factor (EGF)-stimulated Ser473-phosphorylation of Akt was reduced (Fig. 3C; $100 \mathrm{ng} / \mathrm{ml}$ EGF for indicated times) (15). These findings indicate that displacement of KRAS and potentially other tubulin-dependent kinases may yield decreased downstream PI3K signaling, while alternative signaling pathways act on the ERK signaling cascade.

Plinabulin prolongs survival in a RAS-driven glioma model. Next, the specificity of plinabulin effects on KRAS driven types of cancer in a simplified model system that was not biased by co-mutations was determined. For this purpose, two PTEN-proficient glioma gene transfer models that differed solely in the absence or presence of constitutively active KRAS were employed. Gliomas were generated in the brains of Ink4a/Arf-deficient mice expressing the avian t-va receptor under control of the nestin promoter (N/t-va:Ink4a/Arf ${ }^{-/}$) to allow gene transfer utilizing the avian retrovirus RCAS 
A

\begin{tabular}{llll} 
Cell line & Cancer type & KRAS mutation & $\begin{array}{l}\text { in vitro IC } \\
\text { plinabulin }(\mathrm{nM})\end{array}$ \\
\hline HCT-15 & colorectal & G13D & 13 \\
HCT-116 & colorectal & G13D & 33 \\
LoVo & colorectal & G13D & 7 \\
MDA-MB-231 & breast & G13D & 14 \\
RXF-393 & renal & D153V & 4 \\
AsPC-1 & pancreatic & G12D & 17 \\
Capan-2 & pancreatic & G12V & 10 \\
HPAC & pancreatic & G12D & $>1,000$ \\
MIA PaCa-2 & pancreatic & G12C & 92
\end{tabular}

C

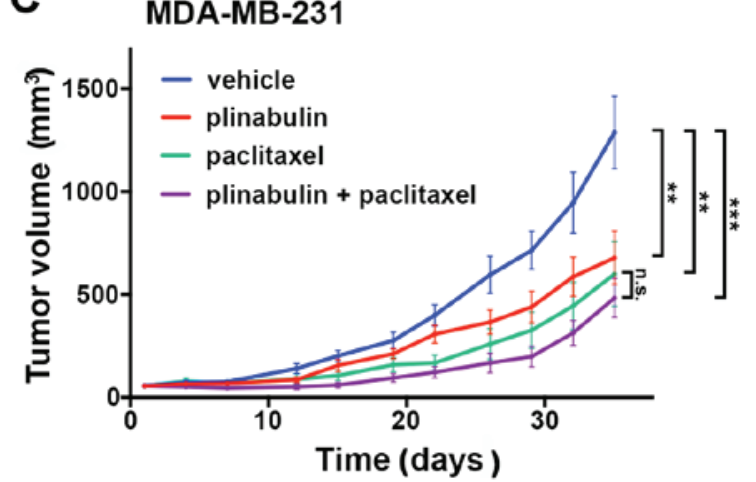

B

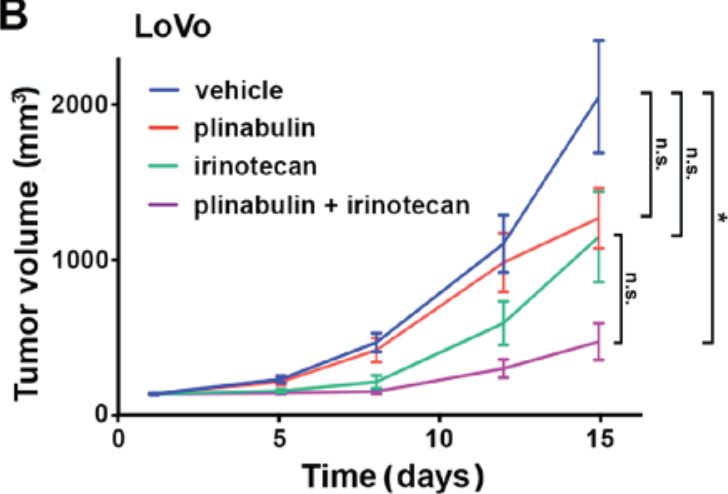

D

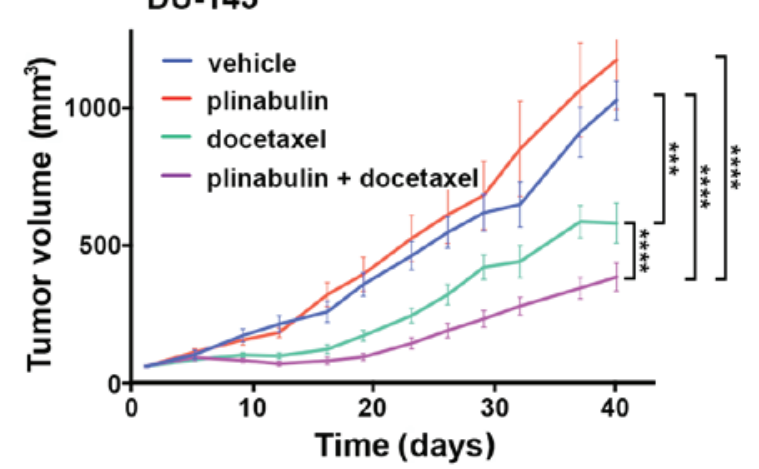

Figure 2. Plinabulin inhibits tumor growth in KRAS mutated cancer models. (A) In vitro growth inhibition of KRAS mutated cancer cell lines by plinabulin. A total of 1,000 cells per well were seeded in 96-well plates in triplicate overnight and treated in serum-free medium with plinabulin at log increments of $1 \mathrm{nM}-16.4 \mu \mathrm{M}$ plinabulin in $0.2 \%$ DMSO or DMSO $0.2 \%$ alone for $72 \mathrm{~h}$ prior to assessment of adenosine triphosphate content. Human KRAS mutated (B) LoVo colon cancer, (C) MDA-MB-231 breast cancer and (D) DU-145 prostate cancer xenograft models were injected as indicated with 5\% dextrose solution (vehicle) i.p. or i.v., plinabulin $7.5 \mathrm{mg} / \mathrm{kg}$ i.p. on 5 subsequent days, (B) irinotecan $80 \mathrm{mg} / \mathrm{kg}$ i.p. once weekly for 3 weeks, (C) paclitaxel $16 \mathrm{mg} / \mathrm{kg}$ i.p. on 5 subsequent days, and (D) docetaxel $12.5 \mathrm{mg} / \mathrm{kg}$ i.v. on days 1,3 and 5 . Tumor volumes were measured twice weekly in $\mathrm{N}=10 \mathrm{mice}$ per treatment group. ${ }^{* *} \mathrm{P}<0.001$ and ${ }^{* * *} \mathrm{P}<0.0001$. i.v., intravenous; i.p., intraperitoneal; NS, not significant.

for overexpression of PDGFB and GFP or KRAS KI2A $^{\text {. }}$ Although activating KRAS mutations are rare in gliomas, hyperactivation of RAS signaling is a key component of gliomagenesis and histologically, these gliomas resemble the human disease (16). Furthermore, given that brain metastases are an important cause of cancer-associated death (17-19), the present study aimed to evaluate whether plinabulin was capable of penetrating the blood brain barrier to a relevant extent. Penetration of the blood-brain barrier by plinabulin is supported by the observation that in a Quantitative Hole Body Autoradioluminogrphy study, plinabulin rapidly entered the brain parenchyma (Fig. S4). Treatment with plinabulin prolonged survival in KRAS-expressing gliomas (Fig. 3D), but not in tumors that were solely driven by PDGFB and Ink4a/Arf loss (Fig. 3E), therefore confirming penetration of the blood brain barrier to a relevant extent and supporting the notion of efficacy of plinabulin specifically in KRAS-mutated tumors.

\section{Discussion}

KRAS-driven cancer poses a major therapeutic challenge. To date, precision medicine approaches have failed to directly target KRAS (4) and standard chemotherapy has only limited efficacy in KRAS-mutated cancer (20). KRAS exerts its oncogenic function primarily by activating the MEK/ERK and PI3K/Akt signaling cascades, but dual inhibition of the two pathways is deemed too toxic to be clinically applicable. However, targeting microtubules has been suggested as a means to compromise endosomal recycling of KRA (21) and combination of docetaxel with the MEK-inhibitor selumetinib dramatically improved the survival of patients with KRAS-driven non-small cell lung cancer compared with docetaxel alone in an exploratory, randomized phase II trial (22)

The authors' preclinical study supports the concept of combining tubulin-targeting agents with inhibitors of the MEK/ERK signaling axis: Upon plinabulin treatment, rapid accumulation of KRAS in endosomal vesicles and a potent inhibitory effect on Akt phosphorylation were observed, along with a simultaneous increase in ERK activation that may compensate for Akt inhibition. The underlying mechanism of this ERK activation likely reflects a stress response through alternative kinases including the stress-activated protein kinases (23).

Compensatory ERK activation may also explain the lack of growth inhibition of most xenograft models tested here by plinabulin monotherapy. Furthermore, effective targeting of KRAS signaling through combined treatment with tubulin-targeting agents and MEK/ERK inhibitors may require intact PTEN, as disinhibition of PI3K signaling upon 
A

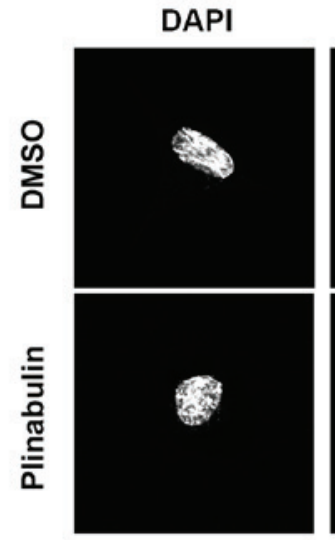

B

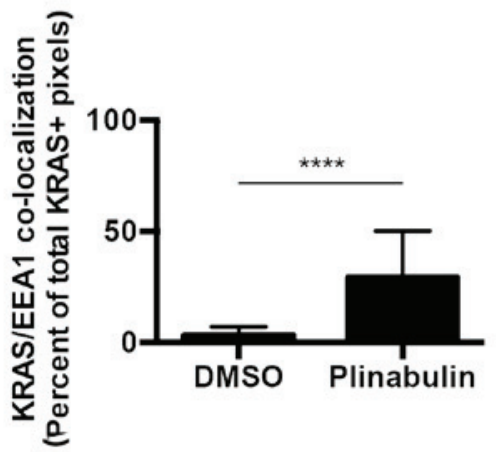

D

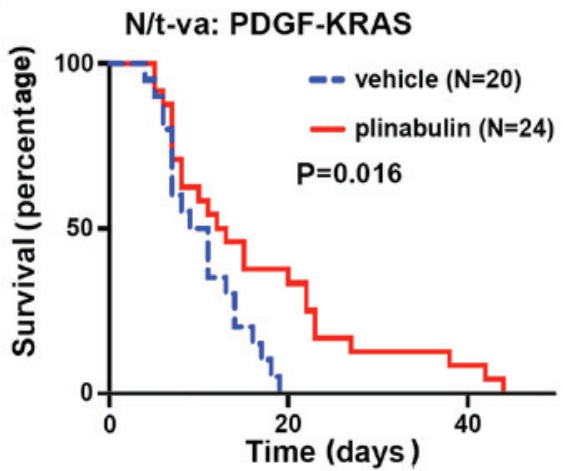

KRAS
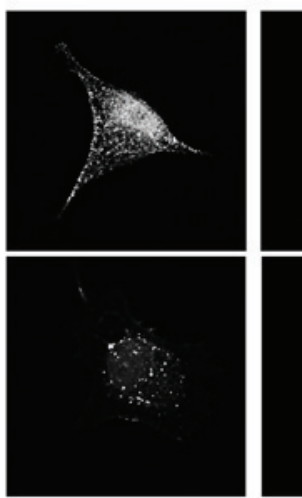

C

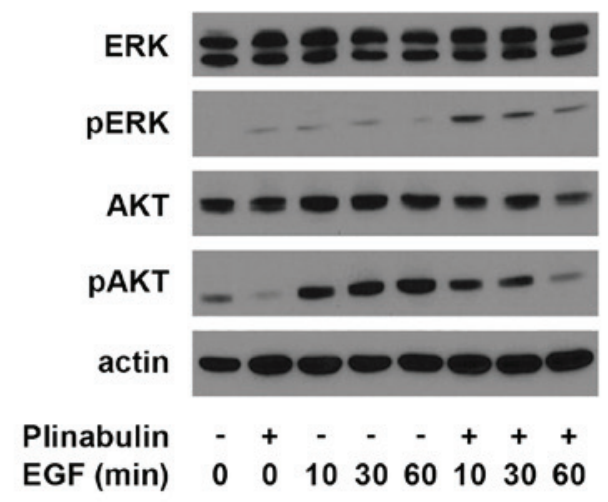

E

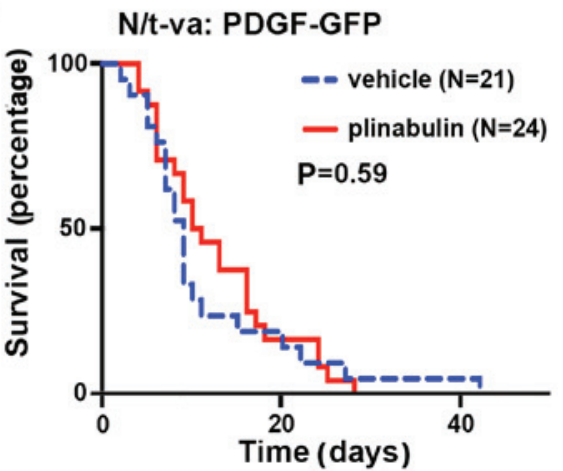

Figure 3. Plinabulin yields endosomal accumulation of KRAS. (A) A549 cells were treated with $0.2 \%$ DMSO (upper panel) or $1.25 \mu$ M plinabulin (lower panel) for $2 \mathrm{~h}$ prior to immunofluorescence staining of DNA utilizing DAPI, (blue), EEA-1 (green) and KRAS (red). Scale bar, $10 \mu \mathrm{m}$. Inlays: $5 \mathrm{x}$ magnification; arrowheads indicate co-localization of EEA-1 and KRAS. (B) Quantification of the percentage EEA1/KRAS double-positive pixels of total KRAS-positive pixels in five high power fields (magnification, x100) from two independent experiments. (C) Immunoblot analysis of A549 cells serum-starved overnight and treated with $1 \%$ DMSO or $1.25 \mu \mathrm{M}$ plinabulin for $2 \mathrm{~h}$ prior to treatment with $100 \mathrm{ng} / \mathrm{ml}$ EGF for indicated times. Mouse gliomas were generated in N/t-va;Ink4a/Arf ${ }^{-/}$mice by transduction with RCAS-PDGF and (D) RCAS-KRAS ${ }^{G 12 A}$, or (E) RCAS-GFP. Mice were treated with 5\% dextrose solution i.p. (vehicle) or plinabulin $7.5 \mathrm{mg} / \mathrm{kg}$ i.p. for 5 subsequent days. Weight loss was utilized as an indicator of tumor formation to determine the time-point of treatment initialization. ${ }^{* * *} \mathrm{P}<0.0001$. EGF, epithelial growth factor; KRAS, Kirsten rat sarcoma viral oncogene homolog; DAPI4, 6-Diamidino-2-phenylindole; ERK, extracellular signal regulated kinase; GFP, green fluorescent protein; PDGF, platelet derived growth factor; DMSO, dimethyl sulfoxide; EEA1, early endosomal antigen 1; pAKT, phosphorylated protein kinase B.

loss of PTEN activity renders Akt signaling independent of KRAS.

Plinabulin may be well suited for such potentially toxic combination treatments, given its favorable safety profile and tolerability in clinical trials. Of note, tubulin polymerization assays reported in the present study indicate that plinabulin exerts its effects by a mechanism that is distinct and potentially less toxic than that of colchicine and established anticancer tubulin-targeting drugs: Plinabulin binds less avidly to tubulin and its binding may be more readily reversible. Furthermore, activity in a mutant KRAS-driven brain tumor model underscores the favorable pharmacokinetics profile of plinabulin, including crossing of the blood brain barrier.

In conclusion, the present preclinical study supports the inclusion of tubulin-targeting agents in future developments of combination treatments against KRAS-driven cancer.

\section{Acknowledgements}

Not applicable. 


\section{Funding}

The present study was funded by BeyondSpring Pharmaceuticals Inc.

\section{Availability of data and materials}

The analyzed data sets generated during the study are available from the corresponding author on reasonable request.

\section{Authors' contributions}

HGW and GL designed the study. Data collection was performed by LH, LD, YW, JB, JDH, KK, PG, JC, SN, MAJ, LW and HGW. LH, LD, YW, JB, JDH, KK, PG, JC, SN, MAJ, LW, PJC and HGW analyzed and interpreted data. PJC and HGW drafted and revised the manuscript. All authors read and approved the final manuscript.

\section{Ethics approval and consent to participate}

All applicable international, national and/or institutional guidelines for the care and use of animals were followed. This article does not contain any studies with human participants performed by any of the authors.

\section{Patient consent for publication}

Not applicable.

\section{Competing interests}

LH, LD, YW and GKL are employed by BeyondSpring Pharmaceuticals Inc. JDH, KK, and PG are employed by Translational Drug Development Inc.

\section{References}

1. Prior IA, Lewis PD and Mattos C: A comprehensive survey of Ras mutations in cancer. Cancer Res 72: 2457-2467, 2012.

2. Fernández-Medarde A and Santos E: Ras in cancer and developmental diseases. Genes Cancer 2: 344-358, 2011.

3. Downward J: Targeting RAS signalling pathways in cancer therapy. Nat Rev Cancer 3: 11-22, 2003.

4. Ostrem JM and Shokat KM: Direct small-molecule inhibitors of KRAS: From structural insights to mechanism-based design. Nat Rev Drug Discov 15: 771-785, 2016.

5. Schmick M, Vartak N, Papke B, Kovacevic M, Truxius DC, Rossmannek L and Bastiaens PIH: KRas localizes to the plasma membrane by spatial cycles of solubilization, trapping and vesicular transport. Cell 157: 459-471, 2014.

6. Thissen JA, Gross JM, Subramanian K, Meyer T and Casey PJ: Prenylation-dependent association of Ki-Ras with microtubules. Evidence for a role in subcellular trafficking. J Biol Chem 272: 30362-30370, 1997.

7. Dumontet $\mathrm{C}$ and Jordan MA: Microtubule-binding agents: A dynamic field of cancer therapeutics. Nat Rev Drug Discov 9: 790-803, 2010
8. Wang Y, Zhang H, Gigant B, Yu Y, Wu Y, Chen X, Lai Q, Yang Z, Chen Q and Yang J: Structures of a diverse set of colchicine binding site inhibitors in complex with tubulin provide a rationale for drug discovery. FEBS J 283: 102-111, 2016.

9. Prota AE, Danel F, Bachmann F, Bargsten K, Buey RM, Pohlmann J, Reinelt S, Lane H and Steinmetz MO: The novel microtubule-destabilizing drug BAL27862 binds to the colchicine site of tubulin with distinct effects on microtubule organization. J Mol Biol 426: 1848-1860, 2014.

10. Mita MM, Spear MA, Yee LK, Mita AC, Heath EI, Papadopoulos KP, Federico KC, Reich SD, Romero O, Malburg L, et al: Phase 1 first-in-human trial of the vascular disrupting agent plinabulin (NPI-2358) in patients with solid tumors or lymphomas. Clin Cancer Res 16: 5892-5899, 2010.

11. Millward M, Mainwaring P, Mita A, Federico K, Lloyd GK, Reddinger N, Nawrocki S, Mita M and Spear MA: Phase 1 study of the novel vascular disrupting agent plinabulin (NPI-2358) and docetaxel. Invest New Drugs 30: 1065-1073, 2012.

12. Ravelli RB, Gigant B, Curmi PA, Jourdain I, Lachkar S, Sobel A and Knossow M: Insight into tubulin regulation from a complex with colchicine and a stathmin-like domain. Nature 428: 198-202, 2004.

13. McCubrey JA, Steelman LS, Chappell WH, Abrams SL, Wong EW, Chang F, Lehmann B, Terrian DM, Milella M, Tafuri A, et al: Roles of the Raf/MEK/ERK pathway in cell growth, malignant transformation and drug resistance. Biochim Biophys Acta 1773: 1263-1284, 2007.

14. Singh A, Greninger P, Rhodes D, Koopman L, Violette S, Bardeesy N and Settleman J: A gene expression signature associated with 'K-Ras addiction' reveals regulators of EMT and tumor cell survival. Cancer Cell 15: 489-500, 2009.

15. Pennock S, Billing S, Wang Z and Wang Y: Two-pulse endosomal stimulation of receptor tyrosine kinases induces cell proliferation. Methods Mol Biol 1652: 127-133, 2017.

16. Uhrbom L, Dai C, Celestino JC, Rosenblum MK, Fuller GN and Holland EC: Ink4a-Arf loss cooperates with KRas activation in astrocytes and neural progenitors to generate glioblastomas of various morphologies depending on activated Akt. Cancer Res 62: 5551-5558, 2002.

17. Langer CJ and Mehta MP: Current management of brain metastases, with a focus on systemic options. J Clin Oncol 23: 6207-6219, 2005.

18. Khuntia D, Brown P, Li J and Mehta MP: Whole-brain radiotherapy in the management of brain metastasis. J Clin Oncol 24: 1295-1304, 2006

19. Chaffer CL and Weinberg RA: A perspective on cancer cell metastasis. Science 331: 1559-1564, 2011.

20. Von Hoff DD, Ervin T, Arena FP, Chiorean EG, Infante J, Moore M, Seay T, Tjulandin SA, Ma WW, Saleh MN, et al: Increased survival in pancreatic cancer with nab-paclitaxel plus gemcitabine. N Engl J Med 369: 1691-1703, 2013.

21. Basseville A, Bates S and Fojo T: Pancreatic cancer: Targeting KRAS and the vitamin D receptor via microtubules. Nat Rev Clin Oncol 12: 442-444, 2015.

22. Jänne PA, Shaw AT, Pereira JR, Jeannin G, Vansteenkiste J, Barrios C, Franke FA, Grinsted L, Zazulina V, Smith P, et al: Selumetinib plus docetaxel for KRAS-mutant advanced non-small-cell lung cancer: A randomised, multicentre, placebo-controlled, phase 2 study. Lancet Oncol 14: 38-47, 2013.

23. Sánchez I, Hughes RT, Mayer BJ, Yee K, Woodgett JR, Avruch J, Kyriakis JM and Zon LI: Role of SAPK/ERK kinase-1 in the stress-activated pathway regulating transcription factor c-Jun. Nature 372: 794-798, 1994.

This work is licensed under a Creative Common Attribution-NonCommercial-NoDerivatives 4.0 International (CC BY-NC-ND 4.0) License. 